\title{
Tratamientos de la colestasis crónica en niños
}

\author{
Fernando Álvarez \\ CHU Sainte-Justine, Universidad de Montreal, Montreal, Que., Canadá
}

\author{
Palabras clave \\ Colestasis crónica • Ácido ursodesoxicólico • Rifampicina • \\ Resinas intercambiadoras de iones - Ácidos grasos $\omega-3 \cdot$ \\ $\mathrm{N}$-acetilcisteína • Antagonista de opioides
}

\section{Resumen}

Se dispone de pocos tratamientos específicos para los niños con colestasis crónica. La mayoría de las estrategias terapéuticas alivian la retención de componentes de la bilis o aminoran algunas de las consecuencias de la colestasis crónica. El ácido ursodesoxicólico es el fármaco que con más frecuencia se utiliza en niños con colestasis crónica. Este ácido biliar se administra a la dosis de 10 a $30 \mathrm{mg} / \mathrm{kg} /$ día en pacientes con fibrosis quística, errores congénitos del metabolismo de los ácidos biliares, colestasis intrahepática familiar progresiva, colangitis esclerosante, atresia biliar, síndrome de Alagille o a aquéllos tratados con nutrición parenteral total. El ácido ursodesoxicólico incrementa principalmente el flujo de bilis y posee un efecto estabilizador de la membrana reduciendo la toxicidad de los ácidos biliares más hidrofóbicos. La rifampicina, un antibiótico, a una dosis entre 10 y 20 $\mathrm{mg} / \mathrm{kg} /$ día, es muy eficaz en el alivio del prurito. Se obtienen efectos similares utilizando resinas intercambiadoras de iones no absorbibles. Además, estas moléculas disminuyen los niveles séricos de colesterol contribuyendo a reducir los xantomas. La sustitución de algunas carencias generadas por la nutrición parenteral total mediante la administración de ácidos grasos esenciales o cisteína puede evitar los trastornos hepáticos asociados o contribuir a mejorarlos. En algunas enfermedades colestáticas, las intervenciones quirúrgicas pueden ayudar a aliviar el obstáculo frente al flujo de bilis, como es el caso de la portoenterostomía en pacientes con atresia biliar. En casos de colestasis interhepática, puede registrarse una mejoría clínica y bioquímica tras una derivación de la bilis u otra intervención (exclusión del íleon), que limita la absorción de ácidos biliares en el intestino. En el futuro, la asociación de estos diferentes agentes farmacológicos, que incrementan el flujo de bilis, protegen las membranas celulares o restablecen las carencias nutricionales, podría contribuir a mejorar la calidad de vida en niños con colestasis crónica y retrasar en última instancia la necesidad de un tratamiento más drástico como el trasplante hepático. También podrían ser de gran ayuda los progresos en la terapia génica y el trasplante de hepatocitos; no obstante, todavía son necesarios muchos años de investigación intensiva antes de que pueda considerarse incluso un estudio piloto que utilice uno de estos tratamientos en hepatopatías inductoras de colestasis crónica.

Copyright $\odot 2009$ Nestec Ltd., Vevey/S. Karger AG, Basel

La colestasis crónica en niños es la consecuencia de una gran diversidad de causas, muy pocas de las cuales tienen tratamiento específico. En la mayoría de los casos se han elaborado tratamientos médicos o quirúrgicos para evitar o tratar las complicaciones consecutivas a la retención de componentes de la bilis. Los tratamientos

\section{KARGER \\ Fax +41613061234 \\ E-Mail karger@karger.ch}

www.karger.com
(C) 2009 Nestec Ltd., Vevey/S. Karger AG, Basel 0252-8185/08/0663-0127\$24.50/0

Accesible online en:

www.karger.com/ans
Prof. Fernando Álvarez, MD

Division of Gastroenterology, Hepatology and Nutrition

CHU Sainte-Justine, University of Montreal

3175, Cote Sainte-Catherine road, Montreal, Qué. H3T 1C5 (Canada)

E-Mail fernando.alvarez@umontreal.ca 
Tabla 1. Tratamientos disponibles para la colestasis crónica en niños

\begin{tabular}{|c|c|c|c|c|}
\hline Tratamiento & Indicación & Contraindicación & $\begin{array}{l}\text { Efectos } \\
\text { secundarios }\end{array}$ & Dosificación \\
\hline AUDC & $\begin{array}{l}\text { Influencias sobre la } \\
\text { patogénesis de CIFP, } \\
\text { FQ y NPT; 'prurito' }\end{array}$ & $\begin{array}{l}\text { Obstrucción de los conductos } \\
\text { biliares (por ejemplo, fallo de } \\
\text { portoenterostomía en } \mathrm{AB} \text { ) }\end{array}$ & Diarrea & 20-30 mg/kg/día \\
\hline Rifampicina & 'Prurito' & Infección por mycobacterium $^{1}$ & & 10-20 mg/kg/día \\
\hline $\begin{array}{l}\text { Resinas intercam- } \\
\text { biadoras de iones } \\
\text { no absorbibles }\end{array}$ & $\begin{array}{l}\text { 'Prurito' } \\
\text { Hipercolesterolemia }\end{array}$ & $\begin{array}{l}\text { Estenosis o suboclusión } \\
\text { intestinal }\end{array}$ & $\begin{array}{l}\text { Estreñimiento } \\
\text { Acidosis } \\
\text { hiperclorémica }\end{array}$ & $\begin{array}{l}8 \text { a } 16 \text { g por vía oral diarios divididos } \\
\text { en } 2 \text { a } 3 \text { tomas (incremento progresi- } \\
\text { vo a partir de } 2 \text { g por vía oral al día) }\end{array}$ \\
\hline $\begin{array}{l}\text { Antagonistas de } \\
\text { opioides }\end{array}$ & 'Prurito' & - & $\begin{array}{l}\text { Reacciones de } \\
\text { abstinencia }\end{array}$ & $?$ \\
\hline Ácidos grasos $\omega$-3 & $\begin{array}{l}\text { Hepatopatía } \\
\text { inducida por NPT }\end{array}$ & - & - & $\begin{array}{l}1 \mathrm{~g} / \mathrm{kg} / \text { día (incremento progresivo a } \\
\text { partir de } 0,2 \mathrm{mg} / \mathrm{kg} / \text { día) }\end{array}$ \\
\hline NAC & $\begin{array}{l}\text { Hepatopatía } \\
\text { inducida por NPT }\end{array}$ & - & - & $\begin{array}{l}70 \text { a } 135 \mathrm{mg} / \mathrm{kg} / \text { día (incremento } \\
\text { progresivo a partir de } 20 \mathrm{mg} / \mathrm{kg} / \text { día) }\end{array}$ \\
\hline
\end{tabular}

Habitualmente son necesarias asociaciones de algunos de estos fármacos. $\mathrm{AB}=$ Atresia biliar.

disponibles son predominantemente de apoyo y tratan de reducir la acumulación de ácidos grasos en el organismo y las consecuencias de dicha acumulación: fibrosis hepática progresiva, prurito, hiperlipidemia y fatiga.

Cantidades menores de ácidos biliares en la luz intestinal son responsables de la malabsorción de grasas. La esteatorrea puede llegar a ser una causa de desnutrición y carencia de vitaminas liposolubles en la colestasis crónica. Algunos tratamientos que se describen más adelante, destinados a evitar la recirculación enterohepática de los ácidos biliares, pueden agravar esta situación.

Agentes farmacológicos como el ácido ursodesoxicólico (AUDC) y la rifampicina mejoran la coleresis incrementando la excreción de ácidos biliares por los hepatocitos. Las resinas intercambiadoras de iones no absorbibles fijan los ácidos biliares en la luz intestinal reduciendo su circulación enterohepática. Todos estos fármacos incrementan la coleresis y disminuyen el prurito y los niveles de colesterol. Aunque se ha propuesto el empleo de antagonistas de opioides para tratar el prurito, su disponibilidad limitada así como sus efectos secundarios restringen su empleo en pediatría (tabla 1).

Las intervenciones quirúrgicas pueden ser curativas, como la intervención de Kasai en algunos pacientes con atresia biliar, o paliativas como la derivación biliar parcial y la exclusión del íleon en pacientes con síndrome de Alagille o colestasis intrahepática familiar progresiva (CIFP).
El último procedimiento podría mejorar el prurito, disminuir la retención de colesterol y evitar la evolución a la cirrosis.

\section{Ácido ursodesoxicólico}

El AUDC (ácido $3 \alpha, 7 \beta$-dihidroxi-5 $\beta$-colan-24-oico) está presente en pequeñas cantidades en la bilis humana, hasta el 3\%, y es el resultado de la $7 \beta$ epimerización del ácido quenodesoxicólico por bacterias colónicas [1].

El grupo $\beta$ hidroxi, más que el grupo $\alpha$ hidroxi, en la $7^{a}$ posición confiere al AUDC una mayor hidrofilicidad cuando se le compara con el ácido quenodesoxicólico, su precursor. Por lo tanto, debido a su menor hidrofobicidad, el AUDC es poco eficiente en la formación de micelas. Además, se absorbe mal en el intestino proximal [1].

\section{Mecanismos de acción}

- Sustitución de ácidos biliares más hidrofóbicos en la reserva circulante y en membranas celulares. Al cabo de 6 meses de tratamiento a la dosis de 10 a $12 \mathrm{mg} / \mathrm{kg} /$ día, el AUDC representa del 40 al 50\% de la reserva de ácidos biliares [2]. Debido a sus propiedades detergentes, los ácidos biliares más hidrofóbicos son tóxicos para las membranas celulares y causan lesión hepatocelular y un aumento de la colestasis. El AUDC posee 
un efecto estabilizador de membrana reduciendo la desorganización de las membranas ricas en colesterol [3].

- El AUDC, pero no su forma conjugada, taurodesoxicolato, disminuye la toxicidad de los ácidos biliares lipofílicos sobre la función de la cadena de transporte de electrones de forma dependiente de la concentración (hasta $100 \mu \mathrm{mol} / \mathrm{l}$ ); sin embargo, a mayores concentraciones se incrementa la toxicidad de las mitocondrias inducida por los ácidos biliares [4].

- Interferencia en el íleon con la absorción de ácidos biliares más tóxicos [2].

- El epitelio biliar reabsorbe el AUDC en forma protonada y lo segrega nuevamente en la bilis (derivación colehepática), dando lugar a un efecto hipercolerético. Los protones absorbidos llevan a la formación de bicarbonato, que es segregado y enriquece la bilis de este componente [2].

- El AUDC incrementa el flujo independiente de sales biliares $[1,2]$.

- El AUDC puede estimular la expresión de trasportadores en la superficie del hepatocito canalicular [5].

- Se ha descrito una reducción de la expresión del antígeno leucocítico humano de clase I en la superficie del hepatocito [6].

- El AUDC también suprime la inducción mediada por el interferón- $\gamma$ de la expresión del antígeno leucocítico humano de clase II a través de la vía mediada por receptores de glucocorticoides [7].

El AUDC es responsable de muy pocos efectos secundarios transitorios, como la diarrea y las reacciones cutáneas, causadas más probablemente por coadyuvantes farmacológicos que por el principio activo. No obstante, debe ser evitado o administrado cautelosamente en pacientes con enfermedad obstructiva del conducto biliar. En animales de experimentación con ligadura del conducto biliar principal, el AUDC puede agravar los infartos biliares [8]. En niños con atresia biliar e intervención de Kasai insatisfactoria, el AUDC puede precipitar una insuficiencia hepática.

El AUDC es el fármaco más frecuentemente utilizado en niños con colestasis crónica. En varias enfermedades, el beneficio de la administración a largo plazo del AUDC no ha sido confirmado más allá de toda duda, como es el caso de la fibrosis quística (FQ). En otros casos, como en pacientes con colangitis esclerosante, la administración del fármaco mejora las pruebas hepáticas, pero no el resultado final. En la CIFP parece detener la progresión de la enfermedad en algunos niños y, asociado al ácido cólico, es el tratamiento de elección en pacientes con errores congénitos del metabolismo de los ácidos biliares. En niños afectados por el síndrome de Alagille o la atresia biliar, el AUDC se utiliza principalmente para reducir síntomas como el prurito.

\section{Fibrosis quística}

$\mathrm{Al}$ cabo de dos meses de tratamiento con AUDC, la presencia de este ácido biliar representa entre el 25 y el $42 \%$ de la reserva, a expensas de los ácidos cólico y quenodesoxicólico; estas diferencias son dependientes de la dosis [9]. A partir de estos estudios se llegó a la conclusión de que la dosificación recomendada de AUDC es de $20 \mathrm{mg} / \mathrm{kg} /$ día o mayor [9].

El AUDC estimula la capacidad secretora biliar en los hígados de pacientes con FQ, tal como se ha determinado por gammagrafía hepatobiliar [10]. En varios estudios piloto se ha observado que el AUDC es responsable de una mejoría en las pruebas bioquímicas, principalmente una reducción de los niveles séricos de aminotransferasas (ALT y AST) y $\gamma$-glutamiltransferasa (GGT) [11]. Además, en las biopsias de control mejoraron la inflamación hepática y/o la proliferación de conductos biliares al cabo de 2 años de tratamiento con AUDC. La administración de AUDC también mejora el estado nutricional de adultos jóvenes afectados de FQ y en niños mejora el estado de los ácidos grasos esenciales y el retinol [12-14]. No obstante, debido a la deficiente actividad del AUDC en la formación de micelas, no influye sobre la esteatorrea [13]. La modificación de la evolución natural de hepatopatías en pacientes con FQ por parte del AUDC sigue pendiente de verificación en un extenso estudio prospectivo y aleatorizado. Recientemente fueron reportados resultados alentadores de detención, o incluso reversión, inducida por el AUDC, de lesiones ecográficas hepáticas observadas en pacientes afectados de FQ en un estudio a largo plazo [15].

\section{Nutrición parenteral total}

El ayuno asociado a la nutrición parenteral total (NPT) da lugar a una estasis de la bilis relacionada con la falta de estimulación del flujo biliar, tanto dependiente como independiente de ácidos biliares. En un modelo de colestasis inducida por NPT, la administración intravenosa de AUDC mejora el flujo de bilis y reduce los niveles de bilirrubina [16]. Se registraron resultados análogamente alentadores en un estudio piloto en niños con diarrea intratable, dependientes de la NPT, pero que toleraban el AUDC a la dosis de $30 \mathrm{mg} / \mathrm{kg} /$ día en 3 tomas diarias [17].

Ann Nestlé [Esp] 2008;66:127-136 
En lactantes con muy bajo peso al nacer y colestasis asociada a NPT, el AUDC a la dosis de 10 a $30 \mathrm{mg} / \mathrm{kg} / \mathrm{día}$ reduce la duración y la intensidad de la colestasis [18]. En la mayoría de estos pacientes, las complicaciones intestinales, incluyendo las resecciones parciales del intestino, impiden el empleo sistemático del AUDC. En última instancia, una dosis diaria muy fraccionada de la medicación puede ser bien tolerada y evitar la diarrea. Desafortunadamente, la forma intravenosa de AUDC no está disponible en el mercado.

\section{Errores congénitos del metabolismo de los ácidos} biliares

De la colestasis crónica en pediatría son responsables dos formas principales de trastorno de la síntesis de los ácidos biliares: la carencia de 3 -oxo- $\Delta 4$-esteroide $5 \beta$-reductasa y la carencia de $3 \beta$-hidro-esteroide- $\Delta 5$-oxidorreductasa/isomerasa. La hepatotoxicidad está causada probablemente por la acumulación de precursores de ácidos biliares y la falta de ácidos biliares primarios. Para reducir la síntesis de moléculas tóxicas debe inhibirse la actividad del colesterol $7 \alpha$ hidroxilasa, la enzima limitadora de la velocidad de síntesis de ácidos biliares endógenos.

La mayoría de los pacientes responden sólo parcialmente al tratamiento con AUDC solo; por tanto, está indicada la asociación con ácido cólico (250 mg/día). En estos pacientes se ha propuesto también la administración de los ácidos quenodesoxicólico y cólico. Este último tratamiento puede dar lugar a una mejoría más rápida de la lesión hepática [19].

\section{Colestasis intrahepática familiar progresiva}

El AUDC es un tratamiento inicial valioso en pacientes con CIFP cuando se administra por vía oral a dosis entre 20 y $30 \mathrm{mg} / \mathrm{kg} /$ día. Se observa una mejoría similar independientemente del tipo de CIFP, es decir, pacientes con niveles séricos de GGT normales (CIFP de tipos 1 y 2) o incrementados (CIFP de tipo 3). En pacientes con GGT normal se observó una mejora de los parámetros de colestasis en el $61 \%$ de los casos y en los pacientes con CIFP de tipo 3, en un 71\% [20]. Estos resultados podrían estar influidos por la duración de la enfermedad antes del comienzo del tratamiento con AUDC; el diagnóstico y el inicio precoces del tratamiento con AUDC podrían mejorar adicionalmente el resultado final. En algunos pacientes, la mejoría, aunque completa, es sólo transitoria y la colestasis reaparece algunos años después del comienzo del tratamiento con AUDC.

En pacientes con menos mutaciones perniciosas en genes responsables de los tipos 1 ó 2 de CIFP se observó una colestasis intrahepática recurrente benigna. En los pacientes afectados por este proceso, el tratamiento con AUDC es incapaz de prevenir un episodio colestático, si bien podría mejorar algunos de los síntomas (por ejemplo, prurito) [21].

\section{Colangitis esclerosante}

En un estudio piloto inicial con AUDC en pacientes con colangitis esclerosante muy desarrollada se observó una mejoría en las pruebas hepáticas. Posteriormente, se observó una diferencia estadísticamente significativa en los niveles séricos de ALT/AST/GGT entre pacientes con colangitis esclerosante tratados con AUDC, o tratados con placebo en el curso de un estudio de 3 años de seguimiento [22]. Aunque también se observó una mejoría del prurito y la fatiga, la diferencia no era significativa. Desafortunadamente, los resultados más recientes de un estudio a doble ciego, aleatorizado, en el que se efectuó una comparación entre AUDC (13 a 15 mg/kg/día) y placebo, no revelaron diferencias en el fracaso del tratamiento (progresión de la enfermedad, fallecimiento o trasplante hepático). Aunque en este estudio se confirmó una mejora de las pruebas hepáticas, se llegó a la conclusión de que el AUDC no ofrecía beneficios clínicos a los pacientes con colangitis esclerosante [23]. En consecuencia, dosis bajas de AUDC no modifican aparentemente la progresión de esta enfermedad. En un estudio publicado muy recientemente se observó que, por el contrario, dosis elevadas de AUDC se asociaban a una mejora de la supervivencia y una tendencia hacia la estabilidad/mejoría del estadio histológico en un grupo relativamente reducido de pacientes con colangitis esclerosante [24].

A pesar de estos resultados, podrían proponerse nuevos ensayos con dosis altas de AUDC, muy precozmente en el curso evolutivo de la colangitis esclerosante y especialmente en pacientes con enfermedad de los conductillos. Es probable que cuando la enfermedad afecta a los conductos biliares principales (tal como acontece en la mayoría de los casos), el trastorno del flujo de bilis pueda contribuir irreversiblemente al empeoramiento de la hepatopatía. La experiencia más extensa con el empleo del AUDC en enfermedades colestáticas crónicas se obtuvo en pacientes adultos afectados de cirrosis biliar primaria, una forma grave de colangiopatía intrahepática [25]. Grupos diferentes mostraron resultados contradictorios en el tratamiento con AUDC, lo que se explica en parte por el momento diferente en la evolución de la enfermedad al comienzo del tratamiento. La dosis de AUDC administrada parece desempeñar también un papel en la influencia de esta medicación sobre el resultado final. Esta vasta 
experiencia debe ser tenida en cuenta en el momento de planificar nuevos ensayos en pacientes con colangitis esclerosante.

\section{Atresia biliar}

En un estudio muy preliminar con AUDC tras portoenterostomía hepática se demostró que este fármaco era eficaz en pacientes con buen drenaje biliar postoperatorio, con el resultado de menores niveles totales de ácidos biliares y una mayor ganancia de peso [26]. La administración de AUDC a largo plazo mejora significativamente el resultado final tras la portoenterostomía de Kasai, más pacientes depuran la ictericia y sobreviven con su hígado original. En niños que no recuperan un buen flujo biliar tras la portoenterostomía, la administración de AUDC puede dar lugar a un desarrollo más rápido de lesión hepática y, por último, precipitar una insuficiencia hepática [27].

\section{Sindrome de Alagille}

Se dispone de pocos trabajos sobre el uso del AUDC en pacientes con síndrome de Alagille. Se registró una mejoría de la ictericia y el prurito, así como una reducción de los niveles de lípidos circulantes y una disminución de los xantomas [28]. Aunque no se dispone de un estudio controlado, el AUDC se prescribe ampliamente en estos pacientes. La influencia de este ácido biliar en la evolución natural de la enfermedad es todavía desconocida.

\section{Rifampicina}

La rifampicina reduce la concentración de ácidos biliares en los hepatocitos por competición con su captación. Además, este antibiótico induce enzimas microsómicas que fomentan la $6 \alpha$-hidroxilación y la subsiguiente $6 \alpha$-glucuronidación de los ácidos biliares tóxicos.

La experiencia clínica inicial con este antibiótico en el tratamiento del prurito procede de adultos con cirrosis biliar primaria; se obtuvo un alivio significativo del prurito en una mayoría de pacientes [29]. En niños con colestasis crónica, un estudio a doble ciego cruzado mostró la eficacia de rifampicina en aliviar el prurito durante un periodo de hasta 6 meses [30]. Subsiguientemente, en dos estudios realizados en pacientes pediátricos con prurito rebelde tratado con rifampicina se confirmaron estos resultados iniciales [31, 32]. En la mayoría de los niños con diferentes causas de colestasis crónica tratados no se registró ninguna mejora de los parámetros bioquímicos de colestasis.

Tratamientos de la colestasis
La dosis recomendada de rifampicina es de 10 a $20 \mathrm{mg} /$ $\mathrm{kg} / \mathrm{día}$ en dos dosis diarias. Se describieron pocos efectos secundarios. No obstante, la rifampicina es un inductor enzimático de enzimas microsómicas. La administración de rifampicina incrementa varias formas de actividad de la isoenzima citocromo P450; en consecuencia, se puede acelerar la velocidad metabólica de diversos fármacos.

Se ha propuesto que el AUDC y la rifampicina producen efectos complementarios que pueden justificar una combinación de ambos agentes en el tratamiento de pacientes con enfermedades colestáticas [33].

\section{Resinas intercambiadoras de iones no absorbibles}

Estas moléculas no son absorbibles en el tubo digestivo, liberan y fijan los ácidos biliares en la luz intestinal disminuyendo su recirculación enterohepática. Las resinas intercambiadoras de iones no absorbibles aumentan la excreción de colesterol a través del incremento de la conversión en ácidos biliares. Estos fármacos pueden disminuir la reserva de ácidos biliares en un $40 \%$ aproximadamente. El ácido biliar trihidroxi se disocia rápidamente de estas moléculas y puede ser absorbido en el íleon.

La colestiramina (gránulos con sabor a naranja) ha sido utilizada durante décadas y ha demostrado su eficacia y seguridad. No obstante, su palatabilidad es mala, lo que limita la tolerabilidad a este fármaco. En consecuencia, en el mejor de los casos el cumplimiento es irregular. Cuando están indicadas sondas nasogástricas para la nutrición enteral, se ha observado que la administración de colestiramina a través de esta vía es bien tolerada y produce efectos satisfactorios en la reducción de los niveles séricos de colesterol y la mejora del prurito (fig. 1).

En niños, el tratamiento con colestiramina puede iniciarse a una dosis de 2 a $4 \mathrm{~g}$ dos veces al día e incrementarse subsiguientemente hasta 8 a 16 g al día en 2 ó 3 dosis divididas. La colestiramina se fija a otros ácidos o a sustancias cargadas negativamente, lo cual explica la mayor parte de su interacción con numerosos fármacos.

El colestipol en gránulos o comprimidos no ha sido utilizado frecuentemente en niños. Sus indicaciones y precauciones de uso son similares a las descritas para la colestiramina.

El clorhidrato de colesevelam (en forma de hidrogel o comprimido no absorbible) es probablemente el mejor tolerado de estos fármacos; sin embargo, desafortunadamente, no está disponible en todas partes [34]. Este fármaco ha incrementado la especificidad, la superior afinidad y la mayor capacidad para fijar ácidos biliares en

Ann Nestlé [Esp] 2008;66:127-136 


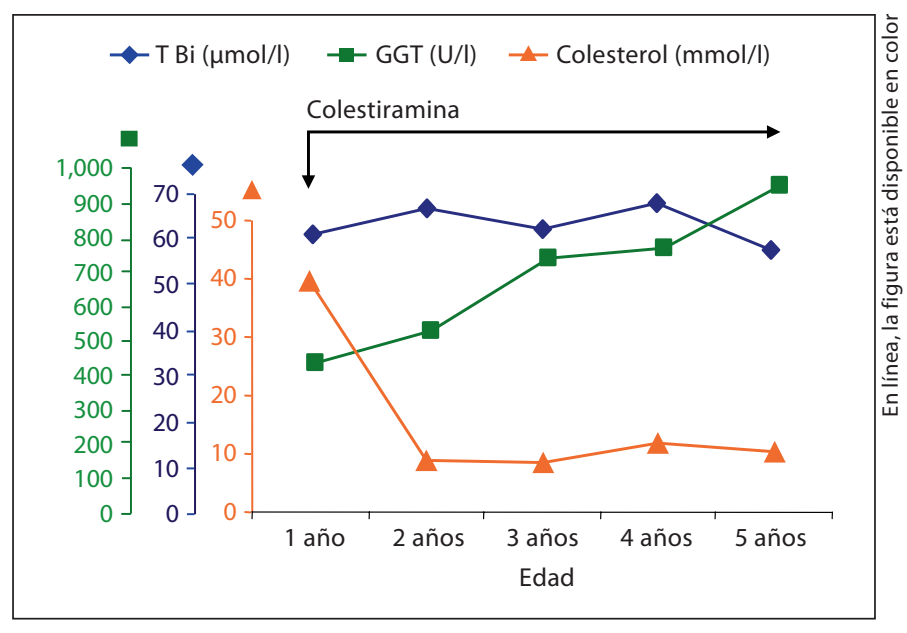

Fig. 1. En este niño con síndrome de Alagille, la colestiramina se administró por sonda nasogástrica, indicada para nutrición enteral. Se observó una reducción espectacular de los niveles séricos de colesterol y la retracción de los xantomas. El prurito mejoró asociando rifampicina al tratamiento. No se registraron modificaciones en los parámetros bioquímicos de colestasis.

comparación con las otras dos resinas intercambiadoras de iones no absorbibles mencionadas anteriormente.

Las resinas intercambiadoras de iones no absorbibles pueden interferir con la absorción intestinal de otros fármacos, como el AUDC. Debe considerarse este problema particular cuando deba tratarse a un paciente con colestasis crónica. Además, el momento de su administración debe establecerse perfectamente para permitir una captación máxima de ácidos biliares y evitar la interacción con otros fármacos incluyendo las vitaminas liposolubles. Otros fármacos deben administrarse 1 ó 4 a 6 horas después de la colestiramina.

Los efectos secundarios principales son estreñimiento y acidosis hiperclorémica debido a las grandes cantidades de cloruro liberadas en la luz gastrointestinal y absorbidas en lugar de bicarbonato. Este último efecto puede ser especialmente grave en pacientes con síndrome de Alagille, que frecuentemente presentan problemas renales previos.

\section{Antagonistas de opioides}

La naloxona y el nalmefeno son los dos primeros antagonistas de opioides ensayados en adultos con cirrosis biliar primaria, en un intento de aliviar el prurito $[35,36]$. La utilización de estas sustancias se basó en el posible papel desempeñado por el sistema nervioso central en el prurito observado en pacientes con colestasis. Las sustancias de la clase encefalina (opioides endógenos) pueden ser mediadores potenciales en la implicación del sistema nervioso.

En un ensayo con naloxona, a simple ciego, controlado con placebo, se demostró que esta medicación reducía la actividad de rascado en pacientes colestáticos. Los problemas principales relacionados con el fármaco consistían en la necesidad de infusiones intravenosas debido a su semivida muy breve y el posible síndrome de abstinencia de opiáceos al final de la infusión [35].

El nalmefeno puede administrarse por vía oral (sin embargo, esta forma no está disponible en todas partes). Se comunicó la mejora de la percepción del prurito en la mayoría de los pacientes tratados, debido a su acción de duración más prolongada en comparación con la de naloxona. Esta medicación puede asociarse a síntomas de abstinencia graves, que dependen aparentemente de la dosis administrada. También se describió una exacerbación notable del prurito cuando el fármaco se suspendía súbitamente [36].

La naltrexona también puede administrarse por vía oral. En un ensayo aleatorizado con naltrexona frente a placebo se comunicó una disminución significativa del prurito diurno y el prurito nocturno. En la mitad de los pacientes tratados con naltroxona se detectó el síndrome de abstinencia de opiáceos [37].

No se ha publicado ninguna serie sobre el uso de antagonistas de opioides en niños con colestasis crónica. En consecuencia, no es posible llegar a una conclusión sobre su indicación en la población pediátrica. A partir de las experiencias con adultos, estos fármacos son difíciles de administrar y es complicado evitar sus efectos secundarios en el momento de su suspensión.

\section{Ácidos grasos $\omega-3$}

La hepatopatía asociada a NPT en niños con insuficiencia intestinal puede dar lugar a cirrosis e insuficiencia hepática. En algunos pacientes, cuando el AUDC se absorbe se registra una mejoría en las pruebas hepáticas. Se obtuvieron resultados más espectaculares sustituyendo los aceites de soja en emulsiones grasas por aceites de pescado. Los niños que recibieron una solución enriquecida con ácidos grasos $\omega$-3 se beneficiaron de una mejoría espectacular de su hepatopatía [38].

Recientemente, un niño de 4 años seguido en nuestro centro fue sometido a una resección de la mayor parte de su intestino delgado debido a un vólvulo. Este niño 
presentó una hepatopatía grave bajo los efectos de la NPT y fue incluido en la lista de espera de trasplante hepático. Se inició un tratamiento con aceite de pescado parenteral (Omegaven); al cabo de 3 meses fue eliminado de la lista de espera y 6 meses después mostraba niveles séricos normales de bilirrubina conjugada [V. Marchand, comunicación personal].

\section{N-Acetilcisteína}

La hepatopatía en la población sometida a NPT a largo plazo es la consecuencia de diversos factores. La agresión oxidativa parece desempeñar un papel principal en la etiología y la persistencia y agravamiento de la colestasis. La concentración celular de glutatión es una defensa esencial frente a este proceso nocivo. La cisteína es un precursor importante del glutatión intracelular; no obstante, niveles bajos de cisteína se encuentran en la solución de aminoácidos actualmente utilizada.

La N-acetilcisteína (NAC) puede utilizarse como precursor de cisteína. Cuando se añadía NAC a dosis de 70 a $135 \mathrm{mg} / \mathrm{kg}$ /día a la solución para nutrición parenteral, se registraba una normalización o reducción notable de los niveles séricos de bilirrubina conjugada [39]. Esta mejoría de la colestasis hepática se asociaba a una normalización del glutatión en los eritrocitos, dando a entender que la concentración celular de esta molécula antioxidante desempeñaba un papel importante en la disminución de la lesión hepática.

\section{Intervenciones quirúrgicas}

\section{Derivación biliar y exclusión ileal}

Hace más de 30 años que Alagille y Odièvre describieron en el Hospital Bicêtre de Francia la eficacia del drenaje biliar por colecistostomía en pacientes con CIFP y síndrome de Alagille. Desafortunadamente, debido a complicaciones locales en la piel en torno al drenaje, este tipo de drenaje biliar externo no podía mantenerse durante mucho tiempo [40].

En la década de los 80 se desarrolló una técnica de derivación biliar parcial mediante $10 \mathrm{a} 15 \mathrm{~cm}$ de yeyuno interpuestos entre la vesícula biliar y la piel. Este procedimiento era permanente y permitía la derivación parcial del flujo de bilis a una bolsa de estoma externo durante años. Se notificó una mejoría clínica y bioquímica en pacientes con síndrome de Alagille [41]. En niños afectados de CIFP, la derivación biliar parcial resultó en mejora del

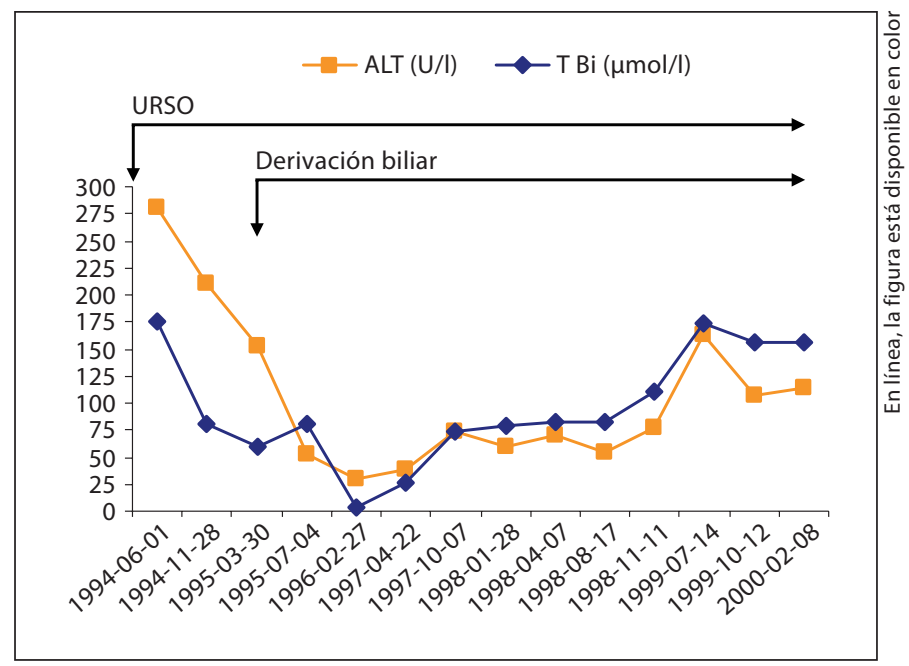

Fig. 2. En esta niña con CIFP (nivel bajo de GGT) se realizó una derivación biliar parcial. Se registró una normalización casi completa de las pruebas bioquímicas hepáticas. Se observó la recidiva de los síntomas y signos de colestasis al cabo de 2 a 3 años de la intervención quirúrgica. La gammagrafía hepatobiliar reveló que el flujo de bilis estaba orientado casi exclusivamente a la bolsa de estoma. Este caso demuestra la progresión de la hepatopatía a pesar de la derivación biliar y el tratamiento con AUDC.

crecimiento, alivio del prurito y corrección de las anomalías bioquímicas de la colestasis [42, 43]. En algunos casos, la mejoría espectacular era transitoria y se seguía de una recaída de la colestasis y la necesidad de un trasplante hepático al cabo de 4 a 5 años de la derivación biliar inicial (observaciones personales) (fig. 2). En otros casos, sólo se obtenía la mejoría completa si se administraba AUDC como tratamiento coadyuvante.

Posteriormente se comunicó una variante técnica en la que se utilizaba el apéndice vermiforme como conducto entre la vesícula biliar y la piel, con resultados muy satisfactorios [44].

En una publicación reciente se describía otro modo de obtener una derivación parcial del flujo de bilis. En tres pacientes con síndrome de Alagille se excluyó el 15\% de íleon terminal utilizando una división engrapada y anastomosis íleocecal. El fundamento de la intervención consiste en evitar la zona de absorción máxima de ácidos biliares en el intestino. Se observó una reducción de los niveles de colesterol, una mejoría del prurito y una reducción de los tamaños de los xantomas [45].

Todas estas intervenciones quirúrgicas deben evitarse en pacientes que hayan presentado ya una cirrosis. Una complicación muy frecuente es la hemorragia copiosa 
procedente de neovarices en las zonas de anastomosis. Además, en pacientes con hepatopatía avanzada no se han demostrado los beneficios de estas intervenciones.

\section{Hepaticoportoenterostomía}

La intervención quirúrgica descrita por Kasai en la década de los 50 llegó a ser la primera y única operación capaz de restablecer el flujo de bilis en pacientes con atresia biliar.

Se comprobó que la normalización de la bilirrubina sérica en el curso de 3 meses a partir de la portoenterostomía indicaba un pronóstico favorable a largo plazo. Esto sucede en alrededor de 50 al 60\% de lactantes operados, con un mejor resultado cuando la intervención se realiza en una etapa precoz de la vida [46]. Otros factores en el momento de la portoenterostomía, como el grado de la fibrosis hepática y las características del residuo extrahepático, pueden modificar el resultado final. El pronóstico de la intervención de Kasai en un paciente con síndrome de malformación poliesplénica asociado a atresia biliar ha sido más desfavorable en algunos centros y similar al de la atresia biliar sin malformaciones en otros [47]. En el periodo postoperatorio, la aparición de colangitis puede también empeorar el pronóstico.

Debido a la presencia de un infiltrado inflamatorio en el hígado de estos pacientes se llegó a suponer que el empleo de fármacos antinflamatorios podría detener la progresión de la enfermedad. Desafortunadamente, varias publicaciones recientes de estudios piloto y estudios a doble ciego aleatorizados revelan que la administración de dosis elevadas de esteroides tras la intervención de Kasai no incrementa la supervivencia del hígado original [48, 49].

La supervivencia a 10 años se sitúa entre el 30 y $54 \%$ en diferentes centros de todo el mundo; alrededor del $25 \%$ a 20 años y del $10 \%$ a 30 años [50-53]. Todos los pacientes presentan cirrosis, con las complicaciones asociadas de hipertensión portal: hemorragia gastrointestinal, hiperesplenismo y enfermedades hepatopulmonares.

El trasplante hepático ha mejorado la supervivencia global de niños con atresia biliar y el fallo de la intervención de Kasai. Actualmente, el éxito de este tratamiento es responsable de una tasa de supervivencia del $90 \%$ [47].

\section{Perspectivas}

Eventualmente, dos tratamientos futuros podrían curar definitivamente algunas enfermedades hereditarias responsables de colestasis crónica: la terapia génica y/o el trasplante de hepatocitos. En el caso de la terapia génica, después de años iniciales de esperanza, los resultados desalentadores obtenidos en la mayoría de los ensayos y los efectos secundarios graves retrasaron su progresión y desarrollo ulterior. En el caso del hígado, se desconoce cuántas células deben expresar un gen específico para evitar la aparición de síntomas y signos de una enfermedad concreta e impedir su progresión. Es preciso resolver numerosos problemas antes de que podamos incluso pensar en un ensayo referente a la hepatopatía colestática.

El trasplante de hepatocitos ha sido ya intentado en algunos humanos. En modelos animales se había observado una corrección parcial de enfermedades hereditarias hepáticas. Los hepatocitos normales poseen una ventaja sobre la repoblación del hígado frente a células portadoras de un gen mutado y posiblemente letal. Se describió la corrección de la CIFP de tipo 3 en un modelo de ratón, que permaneció estable durante un periodo prolongado [54]. Este tratamiento se confronta con la disponibilidad de hepatocitos normales, la necesidad de tratamiento inmunosupresor y la cuestión del destino de las células mutadas residuales. El riesgo de aparición de tumores en el seguimiento a largo plazo representa una preocupación considerable.

Debe explorarse el empleo de ácidos grasos $\omega$-3 en colestasis de orígenes diferentes. Se ha descrito la carencia de ácidos grasos esenciales en pacientes con colestasis de diferentes etiologías, por ejemplo, síndrome de Alagille y FQ. Esta característica es más probablemente una consecuencia de una esteatorrea grave. Aunque se han propuesto vías de administración extraintestinales, su puesta en práctica no ha tenido mucho éxito. La administración intravenosa, periódica, podría estar indicada en algunos casos.

La agresión oxidativa se asocia casi constantemente a la colestasis. Puede plantearse la hipótesis de que, en estos niños, la retención de componentes biliares, la malabsorción responsable de desnutrición y la carencia de ácidos grasos esenciales y oligoelementos da lugar a la malformación de diferentes mecanismos de protección celulares. En la última de las mencionadas, puede incluirse la carencia de glutatión. La administración de NAC como tratamiento coadyuvante podría ser de gran ayuda en varios procesos colestáticos. 
En ensayos futuros debe verificarse el beneficio potencial de los ácidos grasos $\omega-3$ y la NAC en niños con colestasis crónica. Ambos tratamientos muestran muy pocos efectos secundarios y son bien tolerados en niños.

Muchos de los fármacos descritos anteriormente podrían asociarse en el tratamiento de la colestasis crónica con objeto de disminuir la lesión hepática y/o aliviar los síntomas nocivos de estos trastornos. Fármacos con diferentes mecanismos de acción pueden asociarse a un apoyo nutricional adecuado, individualizando el tratamiento con arreglo a la etiología de la colestasis y el estado actual de un niño concreto.

\section{Bibliografía}

$\checkmark 1$ Heuman DM: Hepatoprotective properties of ursodeoxycholic acid. Gastroenterology 1993;104:1865-1870.

-2 Beuers U, Boyer JL, Paumgartner G: Ursodeoxycholic acid in cholestasis: potential mechanisms of action and therapeutic applications. Hepatology 1998;28:1449-1453.

- 3 Heuman DM, Bajaj R: Ursodeoxycholate conjugates protect against disruption of cholesterol-rich membranes by bile salts. Gastroenterology 1994;106:1333-1341.

-4 Krahenbuhl S, Fischer S, Talos C, Reichen J: Ursodeoxycholate protects oxidative mitochondrial metabolism from bile acid toxicity: dose-response study in isolated rat liver mitochondria. Hepatology 1994;20:1595-1601.

- 5 Fickert P, Zollner G, Fuchsbichler A, et al: Effects of ursodeoxycholic and cholic acid feeding on hepatocellular transporter expression in mouse liver. Gastroenterology 2001;121:170-183.

-6 Terasaki S, Nakanuma Y, Ogino H, et al: Hepatocellular and biliary expression of HLA antigens in primary biliary cirrhosis before and after ursodeoxycholic acid therapy. Am J Gastroenterol 1991;86:1194-1199.

7 Tanaka H, Makino Y, Miura T, et al: Ligandindependent activation of the glucocorticoid receptor by ursodeoxycholic acid. Repression of IFN-gamma-induced MHC class II gene expression via a glucocorticoid receptor-dependent pathway. J Immunol 1996; 156:1601-1608

-8 Fickert P, Zollner G, Fuchsbichler A, et al: Ursodeoxycholic acid aggravates bile infarcts in bile duct-ligated and Mdr2 knockout mice via disruption of cholangioles. Gastroenterology 2002;123:1238-1251.

-9 Colombo C, Crosignani A, Assaisso M, et al: Ursodeoxycholic acid therapy in cystic fibrosis-associated liver disease: a dose-response study. Hepatology 1992;16:924-930.

-10 Colombo C, Castellani MR, Balistreri WF, et al: Scintigraphic documentation of an improvement in hepatobiliary excretory function after treatment with ursodeoxycholic acid in patients with cystic fibrosis and associated liver disease. Hepatology 1992;15: 677-684.

11 Colombo C, Setchell KD, Podda M, et al: Effects of ursodeoxycholic acid therapy for liver disease associated with cystic fibrosis. J Pediatr 1990;117:482-489.
12 Lindblad A, Glaumann H, Strandvik B: A two-year prospective study of the effect of ursodeoxycholic acid on urinary bile acid excretion and liver morphology in cystic fibrosis-associated liver disease. Hepatology 1998;27:166-174.

13 Cotting J, Lentze MJ, Reichen J: Effects of ursodeoxycholic acid treatment on nutrition and liver function in patients with cystic fibrosis and longstanding cholestasis. Gut 1990;31:918-921.

14 Lepage G, Paradis K, Lacaille F, et al: Ursodeoxycholic acid improves the hepatic metabolism of essential fatty acids and retinol in children with cystic fibrosis. J Pediatr 1997;130:52-58.

15 Nousia-Arvanitakis S, Fotoulaki M, Economou $\mathrm{H}$, et al: Long-term prospective study of the effect of ursodeoxycholic acid on cystic fibrosis-related liver disease. J Clin Gastroenterol 2001;32:324-328.

16 Duerksen DR, Van Aerde JE, Gramlich L, et al: Intravenous ursodeoxycholic acid reduces cholestasis in parenterally fed newborn piglets. Gastroenterology 1996;111:11111117.

17 Spagnuolo MI, Iorio R, Vegnente A, Guarino A: Ursodeoxycholic acid for treatment of cholestasis in children on long-term total parenteral nutrition: a pilot study. Gastroenterology 1996;111:716-719.

18 Chen CY, Tsao PN, Chen HL, et al: Ursodeoxycholic acid (UDCA) therapy in very-lowbirth-weight infants with parenteral nutrition-associated cholestasis. J Pediatr 2004; 145:317-321.

19 Clayton PT, Mills KA, Johnson AW, et al: Delta 4-3-oxosteroid 5 beta-reductase deficiency: failure of ursodeoxycholic acid treatment and response to chenodeoxycholic acid plus cholic acid. Gut 1996;38:623-628.

20 Jacquemin E, Hermans D, Myara A, et al: Ursodeoxycholic acid therapy in pediatric patients with progressive familial intrahepatic cholestasis. Hepatology 1997;25:519-523.

-21 Crosignani A, Podda M, Bertolini E, et al: Failure of ursodeoxycholic acid to prevent a cholestatic episode in a patient with benign recurrent intrahepatic cholestasis: a study of bile acid metabolism. Hepatology 1991;13: 1076-1083.
22 Stiehl A, Walker S, Stiehl L, et al: Effect of ursodeoxycholic acid on liver and bile duct disease in primary sclerosing cholangitis. A 3 -year pilot study with a placebo-controlled study period. J Hepatol 1994;20:57-64.

-23 Lindor KD: Ursodiol for primary sclerosing cholangitis. Mayo Primary Sclerosing Cholangitis-Ursodeoxycholic Acid Study Group. N Engl J Med 1997;336:691-695.

24 Cullen SN, Rust C, Fleming K, et al: High dose ursodeoxycholic acid for the treatment of primary sclerosing cholangitis is safe and effective. J Hepatol 2008;48:792-800.

25 Corpechot C, Carrat F, Bahr A, et al: The effect of ursodeoxycholic acid therapy on the natural course of primary biliary cirrhosis. Gastroenterology 2005;128:297-303.

26 Nittono H, Tokita A, Hayashi M, et al: Ursodeoxycholic acid in biliary atresia. Lancet 1988;1:528.

-27 Stringer MD, Davison SM, Rajwal SR, McClean P: Kasai portoenterostomy: 12-year experience with a novel adjuvant therapy regimen. J Pediatr Surg 2007;42:1324-1328.

-28 Krawinkel MB, Santer R, Oldigs HD: Ursodesoxycholic acid: effect on xanthomas in Alagille-Watson syndrome. J Pediatr Gastroenterol Nutr 1994;19:476-477.

29 Bachs L, Parés A, Elena M, et al: Comparison of rifampicin with phenobarbitone for treatment of pruritus in biliary cirrhosis. Lancet 1989;1:574-576.

-30 Cynamon HA, Andres JM, Iafrate RP: Rifampin relieves pruritus in children with cholestatic liver disease. Gastroenterology 1990;98:1013-1016.

- 31 Gregorio GV, Ball CS, Mowat AP, Mieli-Vergani G: Effect of rifampicin in the treatment of pruritus in hepatic cholestasis. Arch Dis Child 1993;69:141-143.

-32 Yerushalmi B, Sokol RJ, Narkewicz MR, et al: Use of rifampin for severe pruritus in children with chronic cholestasis. J Pediatr Gastroenterol Nutr 1999;29:442-447.

-33 Marschall HU, Wagner M, Zollner G, et al: Complementary stimulation of hepatobiliary transport and detoxification systems by rifampicin and ursodeoxycholic acid in humans. Gastroenterology 2005;129:476-485.

34 Aldridge MA, Ito MK: Colesevelam hydrochloride: a novel bile acid-binding resin. Ann Pharmacother 2001;35:898-907. 
-35 Bergasa NV, Talbot TL, Alling DW, et al: A controlled trial of naloxone infusions for the pruritus of chronic cholestasis. Gastroenterology 1992;102:544-549.

-36 Bergasa NV, Schmitt JM, Talbot TL, et al: Open-label trial of oral nalmefene therapy for the pruritus of cholestasis. Hepatology 1998;27:679-684.

>37 Wolfhagen FH, Sternieri E, Hop WC, et al: Oral naltrexone treatment for cholestatic pruritus: a double-blind, placebo-controlled study. Gastroenterology 1997;113:12641269.

-38 Gura KM, Duggan CP, Collier SB, et al: Reversal of parenteral nutrition-associated liver disease in two infants with short bowel syndrome using parenteral fish oil: implications for future management. Pediatrics 2006;118:e197-e201.

-39 Mager DR, Marcon M, Wales P, Pencharz PB: Use of $\mathrm{N}$-acetyl cysteine for the treatment of parenteral nutrition-induced liver disease in children receiving home parenteral nutrition. J Pediatr Gastroenterol Nutr 2008;46: 220-223.

40 Alagille D, Odievre M: Les cholestases de l'enfant. Maladies du foie et des voies biliaires chez l'enfant. Paris, Flammarion, 1978.

-41 Emerick KM, Whitington PF: Partial external biliary diversion for intractable pruritus and xanthomas in Alagille syndrome. Hepatology 2002;35:1501-1506.
42 Melter M, Rodeck B, Kardorff R, et al: Progressive familial intrahepatic cholestasis: partial biliary diversion normalizes serum lipids and improves growth in noncirrhotic patients. Am J Gastroenterol 2000;95:35223528 .

43 Kurbegov AC, Setchell KD, Haas JE, et al: Biliary diversion for progressive familial intrahepatic cholestasis: improved liver morphology and bile acid profile. Gastroenterology 2003;125:1227-1234.

44 Gauderer MW, Boyle JT: Cholecystoappendicostomy in a child with Alagille syndrome. J Pediatr Surg 1997;32:166-167.

45 Modi BP, Suh MY, Jonas MM, et al: Ileal exclusion for refractory symptomatic cholestasis in Alagille syndrome. J Pediatr Surg 2007; 42:800-805

46 Ohhama Y, Shinkai M, Fujita S, et al: Early prediction of long-term survival and the timing of liver transplantation after the $\mathrm{Ka}$ sai operation. J Pediatr Surg 2000;35:10311034

47 Chardot C, Serinet MO: Prognosis of biliary atresia: what can be further improved? J Pediatr 2006;148:432-435.
48 Davenport M, Stringer MD, Tizzard SA, et al: Randomized, double-blind, placebo-controlled trial of corticosteroids after Kasai portoenterostomy for biliary atresia. Hepatology 2007;46:1821-1827.

49 Petersen C, Harder D, Melter M, et al: Postoperative high-dose steroids do not improve mid-term survival with native liver in biliary atresia. Am J Gastroenterol 2008;103:712719.

50 McKiernan PJ, Baker AJ, Kelly DA: The frequency and outcome of biliary atresia in the UK and Ireland. Lancet 2000;355:25-29.

51 Chardot C, Carton M, Spire-Bendelac N, et al: Prognosis of biliary atresia in the era of liver transplantation: French national study from 1986 to 1996. Hepatology 1999;30:606611.

52 Shneider BL, Brown MB, Haber B, et al: A multicenter study of the outcome of biliary atresia in the United States, 1997 to 2000. J Pediatr 2006;148:467-474.

53 Schreiber RA, Barker CC, Roberts EA, et al: Canadian Pediatric Hepatology Research Group. Biliary atresia: the Canadian experience. J Pediatr 2007;151:659-665.

54 De Vree JM, Ottenhoff R, Bosma PJ, et al: Correction of liver disease by hepatocyte transplantation in a mouse model of progressive familial intrahepatic cholestasis. Gastroenterology 2000;119:1720-1730. 